\title{
La innovación como estrategia para el desarrollo de los sistemas en la agricultura familiar de Nicaragua
}

\author{
Mauricio Antonio Guzmán Gómez ${ }^{1}$
}

\section{RESUMEN}

El presente artículo es una investigación documental de referencia bibliográfica de tipo no experimental, concerniente al tema de "la Innovación como Estrategia para el Desarrollo de los Sistemas en la Agricultura Familiar de Nicaragua”, publicados con el objetivo de que el lector comprenda y aprenda de este nuevo modelo. Actualmente los países de las Américas enfrenten el desafío común de lograr un desarrollo competitivo y sostenible de la agricultura, que sea compatible con la conservación y el manejo adecuado de los recursos naturales, y con la reducción del hambre y la pobreza en la agricultura familiar. En esencia, hay todo un reto de promover innovaciones, tanto tecnológicas como institucionales para la superación de este flagelo, que tiene que ver con respuestas integrales, mediante alianzas multiactores en los propios territorios. Tomando en cuenta esta problemática, el Gobierno de Nicaragua ha orientado la creación del Sistema Nicaragüense de Investigación e Innovación Agropecuaria SNIA, con el propósito de mejorar la coordinación, compartir información y complementar los procesos de investigación e innovación entre todos los sectores. Sin embargo, hace falta mucho por hacer para empoderar bajo este sistema a las Instiuciones y organizaciones públicas y privadas de que de alguna manera realizan trabajo en los territorios.

Palabras clave: innovación, territorio, sistemas, tecnologías.

Recibido: 28 de julio de 2017

Aceptado: 24 de octubre de 2017

1 Instituto Nicaragüense de Tecnología Agropecuaria (INTA), IV Región. Estudiante del Doctorado Gestión de la Calidad de la Investigación. UNANManagua FAREM-Estelí. Correo electrónico: mauriguz2000@yahoo,com 


\title{
Innovation as a strategy for the development of systems in family farming in Nicaragua
}

\begin{abstract}
This article is a non-experimental bibliographical reference documentary research, related to the theme of "Innovation as a Strategy for the Development of Systems in Family Farming in Nicaragua", published with the objective that the reader understand and learn from this new model. Currently, the countries of the Americas face the common challenge of achieving competitive and sustainable development of agriculture, which is compatible with the conservation and proper management of natural resources, and with the reduction of hunger and poverty in family farming. In essence, there is a challenge to promote innovations, both technological and institutional for the overcoming of this scourge, which has to do with comprehensive responses, through multi-actor alliances in the territories themselves. Taking this problem into account, the Government of Nicaragua has guided the creation of the Nicaraguan System of Agricultural Research and Innovation, SNIA, with the purpose of improving coordination, sharing information and complementing the research and innovation processes among all sectors. However, there is still much more needs to be done to empower under this system the Instiuciones and public and private organizations that somehow do work in the territories.
\end{abstract}

Keywords: innovation, territory, systems, technologies. 


\section{INTRODUCCIÓN}

La innovación, se ha convertido, durante la última década, en un concepto de uso cada vez más frecuente en todo tipo de ámbitos, con el consiguiente riesgo de banalización y utilización meramente ideológica; así ocurre con otros conceptos de perfiles difusos pero que parecen hoy justificar acciones de muy distinto signo (desarrollo sostenible, cohesión, equidad, etc.). No obstante, se ha consolidado entre un buen número de profesionales relacionados con la economía, la gestión empresarial, la sociología o la geografía, la idea de que un esfuerzo de innovación sostenido entendido como la capacidad de generar e incorporar conocimientos para dar respuestas creativas a los problemas del presente, resultahoy unfactorclaveparamejorarla competitividad de las empresas y favorecer un desarrollo en los territorios. Esto no sólo en términos de crecimiento económico, sino desde una perspectiva más integrada. (Méndez, 1998)

En consecuencia, los estudios que incluyen entre sus objetivos prioritarios describir e interpretar los procesos de innovación y sus impactos (económicos, laborales, socioculturales, ambientales, etc.) se han convertido en una de las líneas de investigación más relevantes de los últimos tiempos en ciencias sociales, con especial protagonismo en el ámbito geográfico de las propuestas teóricas y los estudios empíricos que se realizan desde la geografía económica e industrial (Feldman, 1994; Malecki, 1997 y Méndez, 1998).

La nueva concepción de lo rural reconoce una multifuncionalidad del medio rural que va más allá de su papel en la agricultura o de la producción de bienes primarios, se reconoce la capacidad y potencialidad del medio rural para la producción de bienes no agrícolas. Otro aspecto importante de esta nueva concepción es el reconocimiento de la contribución que el medio rural hace al bienestar de la población urbana.

Una definición del medio rural dentro del enfoque de la Nueva Ruralidad es la de Edelmira Pérez : “....El medio rural es un conjunto de regiones o zonas (territorio) cuya población desarrolla diversas actividades o se desempeña en distintos sectores, como la agricultura, la artesanía, las industrias pequeñas y medianas, el comercio, los servicios, la ganadería, la pesca, la minería, la extracción de recursos naturales y el turismo, entre otros. En dichas regiones o zonas hay asentamientos que se relacionan entre sí y con el exterior, y en los cuales interactúan una serie de instituciones, públicas y privadas."

Durante la V Reunión de FORAGRO, realizada en Montevideo 2008, se estableció que: "Los países de las Américas enfrentan el desafío común de lograr un desarrollo competitivo y sostenible de la agricultura que sea compatible con la conservación y el manejo adecuado de los recursos naturales, y con la reducción del hambre y de la pobreza. Enfrentar dicho desafío implica importantes transformaciones, entre ellas las que propician el cambio tecnológico. Particularmente, es necesario desarrollar y compartir una nueva visión del papel de la investigación y la innovación en la agricultura, lo cual implica promover cambios en la institucionalidad de I\&D. En esencia, "hay todo un reto para los países en términos de promover innovaciones, no sólo tecnológicas sino institucionales".

Actualmente la estructura institucional de la investigación agropecuaria de Nicaragua, de acuerdo al informe "Investigación Agrícola Pública en América Latina y el Caribe, Tendencias de Capacidad e Inversión", de Stads y Beintema, 2009, indica que: el INTA alcanza el 33\%; la Universidad Nacional Agraria (UNA), el 45\%; la Universidad Centroamericana (UCA), el 9\%; la Universidad Nacional Autónoma de Nicaragua (UNANManagua), el 8\%; la Universidad de Ciencias Comerciales (UCC), el 4\%; y el Centro para la Promoción, la Investigación, y el Desarrollo Rural y Social, (CIPRES), el 3\%. Sin embargo, aún hace falta una mayor participación, sistémica y coherente de las instituciones públicas, privadas, ONG's, y Universidades, que constituyen agentes importantes para la organización del Sistema Nacional de Ciencia, 
Tecnología e Innovación Tecnológica de Nicaragua, (SINIA), ( Pedroza 2015). (Pedroza \& Enrique, 2015)

Una de las Innovaciones Institucionales más importantes que se están implementando parta modernizar el sector agropecuario Nicaraguense, es la constitución del SNIA el 13 de febrero del 2015, bajo el liderazgo institucional del INTA. El SNIA, es un sistema de actores Institucionales, que aglutina la pluralidad de actores de Investigación, Innovación, Educación, Familias Productoras, Universidades, Sector Privado, ONG's y Cooperación Internacional, etc; de modo que se genere sinergia y complementariedad entre los actores.

El SNIA promueve coordinación horizontal, mutua cooperación, los enfoques Multi, Inter y Transdisciplinarios y la actuación en conjunto de los actores del desarrollo territorial, por medio de un modelo de alianzas, diálogo y consenso entre los actores del desarrollo local; siendo su objetivo general contribuir al desarrollo agropecuario del país, a través del fortalecimiento del modelo de alianzas, consensos y diálogo en materia de investigación e innovación agropecuaria desde los territorios hasta el nivel nacional, en correspondencia con el Plan Nacional de Desarrollo Humano (Pedroza 2016: Conferencia Magistral sobre Innovación Organizacional, presentada en el curso de Gerencia de los Procesos de Inniovación y Emprendimiento).

Sin embargo, hace falta mucho por hacer para empoderar bajo este sistema a todas las Insttiuciones y organizaciones públicas y privadas, que de alguna $\mathrm{u}$ otra manera realizan trabajo en el sector Agropecuario de nuestro país. Por tanto, el presente artículo se basa en la identificación de referencias bibliográficas y electrónicas relacionadas a recabar todo lo concerniente al tema de "La Innovación como estrategia para el Desarrollo Rural Territorial", que contribuya a comprender en el lector, de este nuevo modelo de Innovación Institucional.

\section{MÉTODO}

Uno podrá inventarse todo un mar de excusas, valederas o no, para huir de la mesa de trabajo que nos acecha desde un inoportuno rincón, posponiendo indefinidamente el acto de comunión con la escritura. O decidirse, después de tantas vacilaciones, a enfrentarla, como si uno estuviera sobre un cuadrilátero con ese inasible contendor que es la palabra. Pero al sonar la campana e inaugurarse la hora de la verdad, cuando ya no hay cabida para nuevos aplazamientos, a uno no le queda más remedio que sentarse a escribir.

"Cuando se quiere escribir algo, se establece una especie de tensión recíproca entre uno y el tema, de modo que uno atiza el tema y el tema lo atiza a uno. Hay un momento en que esa relación alcanza un punto ardiente en el que todos los obstáculos se derrumban solos, los conflictos se apartan, y a uno se le ocurren cosas que no había soñado, y entonces, no hay en la vidanada mejor que escribir". Pero ese acto de comunión tan especial tiene, por lo menos, dos momentos: en uno, se pone al espíritu en disposición de entrarle a la escritura, y en el otro, se organiza el material para hacer posible el acto escritural.

Gabriel García Márquez Premio Nobel de Literatura

De ese pensamiento de García Márquez, trata La investigación documental que nos ataña en este artículo, el cual es de tipo no experimental, y basado en una revisión bibliográfica: cuya estructura y esencia que da forma a la investigación es en gran parte la historia, fusionando los principios y métodos de investigación documental; dándonos así, una investigación rica en conocimientos, reflexión e innovación, que a la par nos da elementos para adentrarnos en el estudio del problema, de esta manera llegar a proponer alternativas de solución al problema.

Todo investigador que se adentra en el estudio de los fenómenos sociales, psicológicos, biológicos; tiene que basar su investigación en la historia, ya que es la 
historia de los sucesos, la que nos lleva a cuestionarnos, estudiar, proponer, innovar y aportar nuevos conocimientos, con el fin de seguir construyendo historia y conocimientos; Para llevar a cabo la investigación documental se hace una intensa, rigurosa y larga búsqueda de recolección de información bibliográfica, hemerográfica, electrónica, entre otros. De esta forma la información obtenida se somete a una rigurosa síntesis y análisis de datos, para posteriormente obtener los datos puros que construirán y darán forma al marco teórico.

El objeto que maneja es el documento en su más amplia aceptación, esto es, todo lo que informa, da testimonio o prueba de algo. La recolección de estos datos es estrictamente bibliográfico y también el proveniente de páginas electrónicas. La finalidad de la documentación radica en proporcionar información seleccionada en el menor tiempo posible, el método que utiliza es semejante al de la bibliotecología.

Josefa Sabor, señala que "el ciclo de la documentación es: identificar, organizar y archivar la información para pasar de ahí al de su transformación, síntesis y difusión. En suma, la documentación tiene una función activa, entregar al investigador el material que requiere ya organizado" (Sabor citada en De la Torre \& Navarro, 1990:96).

Tomando en cuenta lo anteriormente descrito, el presente artículo se basa en la identificación de referencias bibliográficas y electrónicas relacionadas a recabar todo lo concerniente al tema de "La Innovación como estrategiapara elDesarrolloRuralTerritorial", que contribuya a comprender al lector de este nuevo modelo de Innovación Institucional.

\section{RESULTADOS DE LA REVISIÓN}

\section{Innovación y Desarrollo Territorial}

En los últimos años se acelera la aparición de nuevos referentes teóricos que sitúan también la innovación y sus relaciones con el territorio como centro de atención, si bien en algunos casos parecerían detectarse ciertos síntomas de rendimientos decrecientes en el plano teórico; no obstante, este tipo de trabajos avanza bastante por delante de la aparición de estudios empíricos, aun claramente insuficientes y realizados con metodologías tan dispares que hacen casi imposible su contrastación.

La propuesta surgida en la llamada Economía del Conocimiento y trasladada al plano territorial con conceptos como región inteligente, learning region o territorios que aprenden (Florida, 1995; Antonelli, y Ferrâo 2001 y Jambes, 2001), todos ellos ligados al actual protagonismo del conocimiento y el aprendizaje colectivo como recursos específicos, es la que parece haber alcanzado una mayor difusión.

El grupo francés sobre Dinámicas de Proximidad (Gilly y Torre, 2000), que centra su atención en la importancia ejercida por la proximidad física además de la funcional y cultural en la creación de redes capaces de transmitir saberes tácitos, no formalizados y difícilmente codificables pero que siguen siendo esenciales para la generación y difusión de innovaciones, aporta una atención específica sobre una temática de tradicional interés geográfico, ahora reinterpretada. Finalmente, los estudios sobre Sistemas Nacionales y Regionales de Innovación (Lundvall y Johnson, 1994 y Cooke y Morgan, 1998) proponen una visión integrada de los procesos innovadores en los que participan actores diversos, desde los que producen conocimiento y lo transmiten a quienes lo utilizan, junto a una serie de instituciones e infraestructuras que regulan ese flujo, lo que permite la elaboración de diagnósticos sobre la estructura del sistema de innovación. (Méndez, 1998)

\section{Innovación y Desarrollo Endógeno Local}

(Brunet y Baltar 2010), toman como referencia que la emergencia histórica de la economía informacional significa, por un lado, que la globalización está dando mayor importancia que antes a los factores que puedan 
estar agrupados bajo la etiqueta de innovación; por otro lado, que hay regiones y localidades que se han transformado en una base fundamental de la vida económica y social mediante redes de cooperación interinstitucional e interempresarial, esto es, redes de organización, producción y gestión institucional territorialmente cooperativa y socialmente enraizada. Redes que explotan los vínculos estratégicos entre la economía de conocimiento intensivo, o informacionalismo, y su insumo estratégico: la innovación (Amin y Thrift, 1994; Storper, 1995). La utilización de estos vínculos es clave para analizar el desarrollo local en la actualidad (Alburquerque, 1996, 1999; Vázquez, 1999). De aquí que el enfoque sobre desarrollo endógeno, apoyándose en la teoría evolucionista (Nelson y Winter, 1982; Freeman, 1998; Edquist, 1997), haya profundizado en las relaciones entre la estructura productiva e institucional para explicar la generación y difusión de innovaciones en una economía, concluyendo que el desarrollo depende de factores internos y concretamente de la calidad institucional de la que estén dotadas las regiones.

El papel que desempeñan las instituciones en el desarrollo territorial estriba en que éste es sensible al contexto institucional, a las condiciones territoriales en las que las empresas realizan sus actividades, en el sentido de que el punto de partida del desarrollo de una comunidad territorial es el conjunto de recursos (económicos, humanos, institucionales y culturales) que constituyen su potencialidad de desarrollo. La capacidad de liderar el propio proceso de desarrollo, unido a la movilización de su potencial de desarrollo "es lo que permite dar a esta forma de desarrollo el calificativo de desarrollo endógeno" (Vázquez, 1999: 30).

De lo anterior se deduce que el desarrollo obedece a la formación de un proceso emprendedor e innovador, con base en la movilización de los recursos específicos y de las propias capacidades de innovación de las regiones (Maillat, 1999; Freeman, 2002; Maskell, 2001; Olazarán y Gómez-Uranga, 2001). Se trata de construir una organización industrial basada en el conocimiento que se traduce en valor económico dentro de un ciclo investigación-desarrollo-produccióncomercialización (Vega-Jurado [et al], 2009).

\section{Innovación Agropecuaria en América Latina}

La innovación en la agricultura ha jugado un papel determinante en el desarrollo económico y social a lo largo de la historia moderna. Fue la innovación en la agricultura lo que posibilitó la liberación de mano de obra para el desarrollo industrial en la Segunda mitad del siglo XIX, así como la consolidación de los mercados para los nuevos productos de los sectores emergentes. Así mismo, la tecnología y la innovación han sido factores determinantes en equilibrar la oferta y la demanda de alimentos a lo largo del siglo XX y un elemento central en desarticular la amenaza maltusiana a pesar de que la población mundial paso en el último siglo de unos 1.650 millones de habitantes a los cerca de 7.000 de la actualidad.

Hitos como la introducción de la genética mendeliana al mejoramiento vegetal a mediados del siglo XIX, los híbridos de maíz en la década de 1930, las variedades de alto rendimiento de la revolución verde y más recientemente los cultivos genéticamente modificados, en la agricultura, y el mejoramiento de pasturas y los avances en la sanidad de la ganadería, son claros y evidentes ejemplos del potencial transformador de la innovación y los inmensos beneficios sociales y económicos asociados a esos procesos. (Trigo, Mateo, Falconi, 2013).

\section{La innovación y su importancia para el crecimiento económico sostenible de Nicaragua}

La economía mundial actual evidencia que el conocimiento científico y tecnológico, y la innovación, son factores claves para un crecimiento económico sostenible. Gran parte de las ventajas competitivas que lospaíses ostentanhoyendía derivadel aprovechamiento del conocimiento científico convertido en tecnología y 
aplicado en el plano empresarial a desarrollar nuevos productos o nuevos servicios, a través de la gestión de procesos de innovación tecnológica, de comercialización, y organizativa.

La globalización mundial pone de manifiesto la urgente necesidad de que las organizaciones empresariales que producen bienes y servicios para el mercado doméstico o internacional, reconozcan el valor estratégico de la innovación y la incorporen en su gestión empresarial como un instrumento o herramienta que forme parte de su cultura corporativa. Es importante que la empresa nicaragüense, en especial la MIPYME (micro, pequeña y mediana empresa), reconozca que factores claves como: Productividad, competitividad, mercadeo eficiente y eficiencia organizativa, dependen cada vez más de implementar mecanismos idóneos que permitan pasar de la etapa de generación del conocimiento (Cienciay Tecnología), o de la importación y asimilación del mismo, a la etapa de su aplicación práctica mediante los procesos de innovación.

Efectuar el tránsito de la Ciencia y Tecnología, o de la Investigación y Desarrollo (I+D), a la innovación empresarial, hoy día constituye uno de los retos más importantes que enfrentan los países en desarrollo. Para Nicaragua esto debe ser un esfuerzo integral que involucre a sectores y actores estratégicos. Por ello, la Universidad, con sus centros de investigación y desarrollo, yelGobierno, consus limitadas posibilidades de financiamiento y asistencia técnica, aisladamente no podrían lograr el crecimiento económico sostenible del país. Se requiere también del proceso productivo empresarial, incluyendo a las MIPYMES, a fin de participar exitosamente en mercados cada vez más competitivos.

La mayoría de los países reconocen la importancia de la Ciencia y la Tecnología como herramientas para el crecimiento económico, pero muchos de los países en desarrollo se enfocan exclusivamente en la generación del conocimiento en apoyo a la investigación básica. Un enfoque pragmático para Nicaragua, debe concebir la inversión en Ciencia y Tecnología, a través de la Investigación y Desarrollo (I+D), no como un fin en sí misma, sino por su contribución a la producción de bienes y servicios en mayor cantidad y calidad, a reducir la pobreza a través del aumento del empleo, y en general, a elevar el nivel de vida de la población.

A pesar de que Nicaragua cuenta con un Consejo de Ciencia y Tecnología (CONICYT), con funciones determinadas por Decreto Presidencial para promover la Ciencia y Tecnología, y con su propio presupuesto operativo, esta entidad ha carecido de una visión y vinculación útil para ayudar a resolver los problemas del crecimiento y desarrollo concretos a nivel productivo. Por más amplia que sea su representatividad de miembros, carece de una vinculación adecuada con las instancias que definen las políticas económicas de este país, por lo que no ha podido desarrollar propuestas inteligentes para la gestión de políticas, programas y proyectos que incorporen la Ciencia, Tecnología e Innovación para la solución de los problemas que enfrentamos todos los nicaragüenses, a diario.

Nicaragua necesita con urgencia formular, discutir y consensuar con todos los sectores estratégicos, una Política de Ciencia, Tecnología e Innovación, con su correspondiente Plan Estratégico, y eventualmente aprobarla e implementarla. Dicha Política debería proponerse alcanzar tres objetivos mediante la incorporación del conocimiento, para resolver problemas sociales y productivos fundamentales del país: 1) Cumplir las metas del Milenio incorporando soluciones innovadoras a los problemas de agua, luz, alcantarillado, etc; 2) Incrementar la productividad de las empresas del sector agropecuario mediante asistencia técnica, semillas mejoradas, riego por goteo, así como otras soluciones innovadoras; y 3) Incrementar la productividad y diversificación de productos con mayor valor agregado de empresas del sector industrial. (Regina Lacayo Oyanguren*) 


\section{Desarrollo Rural Territorial}

(Rojas y Espinoza 2013), en su libro Desarrollo Rural Territorial, mencionan que nuestro país a lo largo de los últimos cincuenta años ha estado influenciado por distintos paradigmas del desarrollo rural, los cuales en menor o mayor medida han normado las políticas públicas. Una de las lecciones más importantes que nos deja este caminar, es que el fenómeno de la pobreza rural es complejo y que las posibilidades de avanzar hacia la superación de este flagelo, tiene que ver con respuestas integrales, multidisciplinarias y mediante alianzas multiactores en los propios territorios.

En este contexto, sobresale el planteamiento de un nuevo enfoque del desarrollo rural, integrador de espacios, actores, políticas, y mercados. Esto implica una serie de desafíos en términos de crear mecanismos para generar, gestionar y compartir conocimientos al interior de los territorios - entre agricultores, el sector público, investigadores y centros especializados.

El deterioro de los recursos naturales, migración rural, adaptación al cambio climático y persistencia de la pobreza plantean la necesidad de construir procesos de desarrollo endógeno, basados en experiencias innovadoras de desarrollo. Un desafío urgente es aprender de las experiencias de los procesos de desarrollo territorial para construir nuevos enfoques y perspectivas. Tales argumentaciones, dan paso a la pertinencia que podrían estar jugando las redes de gestión del conocimiento en la incorporación de saberes en los procesos de aprendizajes colectivos, y la actitud innovadora.

\section{Un Nuevo Modelo de Innovación Tecnológica para el Agro Nicaragüense}

(Pedroza, 2010), señala diferentes puntos de vistas del concepto de Innovación de algunos funcionarios e instituciones que se mencionan a continuación:
Innovación Tecnológica, de acuerdo al PNTFTA 2002, es conjunto de procesos desde la generación hasta la adopción de tecnologías, en función de las necesidades e intereses de agricultores y empresarios agrícolas, dirigidos a mejorar la competitividad de sus sistemas de producción y comercialización. Innovación Tecnológica, de acuerdo a Samper M., 2004, se refiere principalmente a la introducción de cambios en los sistemas agropecuarios, desde el cultivo, cría o aprovechamiento primario de los recursos naturales hasta la recolección, el manejo post-cosecha y la transformación de los productos, con el fin de mejorar, cuantitativa o cualitativamente, la producción.

La Innovación Tecnológica es un proceso tanto material como social, en el cual se aplican conocimientos adquiridos por diversas vías y se toman decisiones sobre el manejo de la unidad productiva. Este proceso requiere del aprendizaje por diversas vías y del intercambio espontáneo u organizado de conocimientos entre agricultores y agricultoras, como también directa o indirectamente, con el personal técnico y científico.

La innovación tecnológica, en la agricultura campesina, pone en contacto al conocimiento local que combina herencias, ensayos y otros aprendizajes, con el saber científico-técnico. La comunicación misma es un proceso de construcción social de conocimientos, en este caso, mediante el diálogo entre lo que saben los experimentadores campesinos u otros miembros de sus comunidades y lo que aportan los extensionistas u otros especialistas.

Innovación Tecnológica, de acuerdo a REDSICTA, 2005, es la adopción de cambios en el ámbito de los productos, procesos, comercialización y formas de gestión en cualquier punto de las cadenas agroalimentarias, a una escala que tenga significación

Innovación Tecnológica, de acuerdo al Dr. Julio Santamaría, 2005, citando su obra Escenarios futuros para la tecno ciencia y la innovación agropecuaria y forestal en Panamá, innovar en un sentido más amplio, 
se refiere a "crear una nueva forma de hacer las cosas". Innovar va más allá de fabricar y lanzar productos avanzados. Innovar es intentar trabajar de manera más inteligente, enfrentándose a los desafíos desde nuevos ángulos, imaginando fórmulas para mejorar las cosas.

Innovación Tecnológica, de acuerdo a la Comisión Interinstitucional de Innovación Tecnológica, INTAMAGFOR- FUNICA, 2008, es un proceso sistemático de generación, modificación, adaptación, disposición y aplicación de nuevas tecnologías, aplicadas en diferentes actividades socio productivas, ambientales e institucionales, para satisfacer las necesidades propias del desarrollo humano

Innovación Tecnológica, de acuerdo al Dr. Sergio Salles Filho, 2008, citando el Manual de Oslo: Es el momento en el cual se verifica la apropiación social (vía mercado o no) de productos, servicios, procesos, métodos y sistemas que no existían anteriormente, o con alguna característica nueva y diferente de la vigente.

El M.A. Mario R. López, 2009, Director del CINETFacultad de Ciencias Económicas, UNAN-Managua, señala que la innovación tecnológica, es un proceso social, interactivo, complejo y ubicuo. El mismo autor, enfatiza que el concepto de innovación tecnológica se usa como sinónimo de "nuevas combinaciones", y lo que se combina en la innovación son: diversas piezas de conocimientos. El mismo autor refiere que, por definición innovación tecnológica implica la creación y aplicación de nuevas cosas y .... nuevo conocimiento, cualitativamente diferente.

Finalmente destaca tres tipos de innovación: a) Innovación de Productos, (nuevos bienes y servicios); b) Innovación Organizacional e Institucional; c) Innovación de Procesos, (procesos tecnológicos, procesos organizacionales).

Sigue diciendo (Pedroza, Henry, 2010), en su libro Un Nuevo Modelo de Innovación Tecnológica para el Agro Nicaragüense, que la estrategia, tiene por objetivo mejorar el bienestar de las personas y comunidades más empobrecidas, es decir que ayude a superar obstáculos y ampliar las oportunidades de éstas, constituyéndose en medio para promover las capacidades de la gente y su autonomía, (www.conicyt. gob.ni).

Está claro entonces, que la innovación tecnológica debe entenderse como una respuesta necesaria y estratégica para superar la problemática de baja adopción tecnológica y baja productividad en Nicaragua. La innovación tecnológica, es un componente fundamental de la competitividad en cualquier sistema de producción, por lo que facilita desarrollar la competitividad en el agro nicaragüense. Por lo tanto, la innovación tecnológica contribuye a resolver, por un lado, el creciente problema de pobreza rural y urbana e inseguridad alimentaria, y por otra parte, a competir en los mercados locales e internacionales para generar las divisas necesarias que demanda el desarrollo humano sostenible de los y las nicaragüenses.

Durante la conferencia internacional "Nicaragua Innova !!!”, con el apoyo de ASDI, (Agencia Sueca para el Desarrollo Internacional), realizado el 6 y 7 de julio de 2010, bajo el lema "Juntando esfuerzos para el desarrollo...", la Lic. Regina Lacayo, representante de CACONIC (Cámara de Comercio de Nicaragua), en su conferencia "La innovación desde la perspectiva industrial”, presentó el concepto de innovación tal como: "Innovar es crear o mejorar productos y servicios para facilitar la vida del cliente ..." Por otra parte, desde la perspectiva CONICYT, expuesta en el "Premio Nacional a la Innovación para el desarrollo Humano", el concepto de Innovación es: "Un nuevo producto, método, técnica o estrategia, obtenido por cambios inducidos en lo esencial o particular del mismo, pero con características similares al original, para solucionar un problema o suplir una necesidad de la sociedad en general y con el cual se disminuyen costos, se aumentan la eficacia y la eficiencia del mismo". 
Un Nuevo Modelo de Innovación Tecnológica para el Agro Nicaragüense estrategia, tenga por objetivo mejorar el bienestar de las personas y comunidades más empobrecidas, es decir que ayude a superar obstáculos y ampliar las oportunidades de éstas, constituyéndose en medio para promover las capacidades de la gente y su autonomía, (www.conicyt. gob.ni).

Está claro entonces, que la innovación tecnológica debe entenderse como una respuesta necesaria y estratégica para superar la problemática de baja adopción tecnológica y baja productividad en Nicaragua. La innovación tecnológica, es un componente fundamental de la competitividad en cualquier sistema de producción, por lo que facilita desarrollar la competitividad en el agro nicaragüense. Por lo tanto, la innovación tecnológica contribuye a resolver, por un lado, el creciente problema de pobreza rural y urbana e inseguridad alimentaria, y, por otra parte, a competir en los mercados locales e internacionales para generar las divisas necesarias que demanda el desarrollo humano sostenible de los y las nicaragüenses

IICA (1992), plantea que "dado el patrón de desarrollo tecnológico que ha predominado en Centro América no ha seguido un enfoque dirigido a la sostenibilidad, se hace necesario la integración de diferentes enfoques, para que surja un paradigma de sostenibilidad basado en la ecología, con los ecosistemas como centro de atención (el ser humano como parte central del ecosistema), con un enfoque de sistemas, con una Investigación de tipo participativa, observando en primer grado las interacciones entre los elementos, favoreciendo la diversificación, buscando la optimización de la "productividad" del sistema (todos los productos y servicios), y mantener el capital ecológico".

El Banco Mundial (2006), destaca que, debido al mayor énfasis en el impacto logrado, las estrategias de ciencia y tecnología agrícola han pasado, en la última década, de la perspectiva de un Sistema Nacional de Investigación Agrícola (SNIA), a la de un Sistema de Conocimiento e Información Agrícola (SCIA) y, más recientemente, a la de un Sistema Nacional de Innovación Tecnológica Agrícola (SNITA).

Por su parte, Alarcón E, (2008), sintetiza la evolución organizacional que han vivido en América Latina las instituciones de ciencia y tecnología -INIAS-: Básicamente, en el contexto institucional actual, desde los años 1960 hasta la fecha, hay una clara transición del paradigma de GTTA (Generación y Transferencia de Tecnología Agropecuaria), hacia el paradigma de Innovación Tecnológica.

Un Nuevo Modelo de Innovación Tecnológica para el Agro Nicaragüense basado en el Modelo $\mathrm{I}+\mathrm{D}+\mathrm{i}$. (Pedroza, 2006), Estrechamente vinculado al concepto de innovación se encuentra el concepto de Sistema de Innovación Tecnológica. Por su importancia abordamos aquí el concepto citado en la obra Enhacing Agricultural Innovation, ¿How to go beyond the strengthening of research systems?, (Banco Mundial, 2007): el concepto de sistema de innovación puede definirse como "una reddeorganismos, talescomoindividuos, organizaciones y empresas, orientadas a iniciar el uso económico de nuevos productos, nuevos procesos y nuevas formas de organización, junto con las instituciones y políticas que afectan su comportamiento y desempeño. Los sistemas de innovación se enfocan, no sólo en aquellos que lleva a cabo la ciencia, sino también en la totalidad y la interacción de los actores participantes en la innovación. La aplicación de este concepto al sector agrícola presenta oportunidades de expandir la base de conocimientos y brinda opciones para desarrollar nuevas tecnologías agrícolas.

Por su parte, Salles et al 2009, destaca el momento actual de la evolución del modelo de GTTA en América Latina, señalando como premisa síntesis que: "La agenda de investigación, más que investigación, debe ser una agenda de innovación”. Continúa señalando que la agenda de innovación debe considerar las 
heterogeneidades: a) Perfil de productores; b) Geográficas; c) Culturales; d) Socioeconómicas; e) Ambientales. Finaliza afirmando que: "No hay un modeloúnico, pero hay elementos comunes y elementos específicos". El mismo autor, basado en los temas claves a implementar para el desarrollo de la región LAC, considera como la premisa número 1 que "los temas claves no son apenas de investigación", ya que más bien "son de innovación", lo que implica la participación de múltiples actores, y significa que se deben incluir múltiples perspectivas, que incluye la producción, comercialización, escalamiento, propiedad intelectual, asistencia técnica, distribución, etc. (Pedroza, 2010)

\section{Instalación SNIA en Nicaragua}

Tomando en cuenta todo lo anteriormente descrito, El Gobierno de Reconciliación y Unidad Nacional le ha asignado al INTA la coordinación del Sistema Nicaragüense de Investigación e Innovación Agropecuaria SNIA, el cual tendrá como propósito mejorar la coordinación, compartir información y complementar los procesos de investigación e innovación entre productores, productoras, universidades, centros e institutos públicos y privados de investigación. La creación del SNIA expresa el modelo de alianzas, consensos y diálogo permanente, promovido por el Presidente Comandante Daniel Ortega. En este caso el modelo se aplicará en la investigación e innovación agropecuaria como instrumento prioritario para avanzar en la mejora de la productividad agropecuaria, la seguridad alimentaria y nutricional y el cuido a la madre tierra.

E1 Sistema Nicaragüense de Investigación e Innovación Agropecuaria, se define como un mecanismo de concertación, coordinación, planificación, implementación, seguimiento y evaluación de la actividad de investigación e innovación agropecuaria del país, que tiene como propósito contribuir al desarrollo de la capacidad productiva de productor@s.
El SNIA está integrado por un Consejo Nacional de Investigación e Innovación Agropecuaria, Consejos Regionales de Investigación e Innovación Agropecuaria y los Núcleos de Investigación e Innovación Territorial, y tiene por objetivo general Contribuir al desarrollo agropecuario del país, a través del fortalecimiento del modelo de alianzas, consensos y diálogo en materia de investigación e innovación agropecuaria desde los territorios hasta el nivel nacional, en correspondencia con el Plan Nacional de Desarrollo Humano. (Pedroza y Mendieta 2015)

Riesgos en la conformación de un SNIA

Según De Sousa 2004, citado por (Pedroza, 2015), la conformación de un SNIA tiene algunos riesgos en su conformación y que se mencionan a continuación:

1. Ineficiente uso de los recursos por el predominio de agendas dispersas, investigación desarticulada y duplicada, con escasas alianzas funcionales.

2. El cortoplacismo y el parcial cubrimiento de las demandas del sector.

3. Carencia de un sistema de información que contribuya a articular el sistema con la demanda y la oferta tecnológica.

4. Brechas amplias entre desarrollo tecnológico e innovación.

5. Alta endogamia del conocimiento y baja interacción entre sistema y el conocimiento global.

6. Limitado trabajo en red.

7. Esporádica evaluación de impacto (ex-ante y expost).

\section{CONCLUSIONES}

De toda esta revisión bibliográfica presentada en este artículo relacionada a "La Innovación como Estrategia para el Desarrollo Rural Territorial en Nicaragua”, podemos deducir que en La Región de America Latina y en especial Nicaragua, han avanzado considerable en este campo de La Innovación y el Desarrollo Rural, sustentados por todos los articulos, libros, revistas, 
tesis, documentos en lineas que sus autores publicaron, basados en un estudio exhaustivo de estos temas que con mucho ainco y dinamismo fueron escrito con un objetivo en común:

La creación de un espacio en donde se conjuguen las experiencias, conocimientos y compromisos que los distintos sectores de la región tienen y que necesitan depositar como elementos de una agenda innovadora y banco de información, que sirva de guía para direccionar el trabajo de aquellos gestores del desarrollo regional que esperan que su acción consciente trascienda en impactos y bienestar para las familias campesinas y para la sociedad civil de nuestra región y en especial de nuestro país.

El solo hecho de conocer que El Gobierno de Reconciliación y Unidad Nacional oriento y ejecuto la creación del Sistema Nicaragüense de Investigación e Innovación Agropecuaria SNIA, el cual tendrá como propósito mejorar la coordinación, compartir información y complementar los procesos de investigación e innovación entre productores, productoras, universidades, centros einstitutos públicos y privados de investigación. La creación del SNIA expresa el modelo de alianzas, consensos y diálogo permanente, promovido por el Presidente Comandante Daniel Ortega. En este caso el modelo se aplicará en la investigación e innovación agropecuaria como instrumento prioritario para avanzar en la mejora de la productividad agropecuaria, la seguridad alimentaria y nutricional y el cuido a la madre tierra.

Sin embargo, hace falta mucho por hacer para empoderar bajo este sistema, a las Insttiuciones y organizaciones publicas y privadas, que de alguna $\mathrm{u}$ otra manera realizan trabajo en el sector Agropecuario de nuestro país, basado en De Sousa 2004, en que la conformación de un SNIA tiene algunos riesgos en su conformación y el mismo De Sousa 2012, menciona que: Un sistema socio-técnico no existe, hasta que la mayoría que lo integra inicia a pensar y a actuar como si fuera un sistema. Sólo entonces el sistema empieza a ganar una dinámica parecida con la dinámica de un sistema.

En consecuencia, se recomienda implementar en todas las regiones y territorios del País, El Sistema Nicaragüense de Investigación e Innovación Agropecuaria SNIA, como una herramienta metodológica de concertación, coordinación, planificación, implementación, seguimiento y evaluación de la actividad de investigación e innovación agropecuaria del país, que tenga como propósito contribuir al desarrollo de la capacidad productiva de productores.

\section{REFERENCIAS BIBLIOGRÁFICAS}

CONICYT, (2010). Plan Nacional de Ciencia y Tecnología de Nicaragua. Managua, Nicaragua

De Sousa J. (2005) "La Innovación de la Innovación Institucional”: de lo universal, mecánico y neutral, a lo contextual, interactivo y ético, desde una perspectiva Latinoamericana.

Eduardo Trigo E. Mateo N. y Falconi C. (2013) "Innovación Agropecuaria en América Latina y el Caribe”: Escenarios y Mecanismos Institucionales Falconi, C. y Elliott, H. (1994). "Investigación agrícola y el sector privado": hacia un marco conceptual.

FORAGRO. (2008). “Declaración de Montevideo.”: V

Foro de Tecnología: Innovaciones institucionales para una agricultura con conocimientos, en las Américas del siglo XXI.

IgnasiBrunet Icarty Fabiola Balta.r(2010). "Desarrollo endógeno, calidad institucional e innovación”. Una revisión de la teoría y de algunos de sus límites*

IICA. (1992). "Tecnología y sostenibilidad de la agricultura en América Latina”: Desarrollo de un marco conceptual-. San José C.R. 132 p.

López M. (2013) "Agroindustria y Sistemas de InnovaciónenNicaragua"Centrodeinvestigaciones Económicas y Tecnológicas CINET - UNAN Docente titular Departamento de Economía Agrícola DEA UNAN- Managua

Méndez, R. (1998). “Innovación y desarrollo 
territorial": algunos debates teóricos recientes.

Pedroza, H., (2010) "Un nuevo modelo de innovación tecnológica para el agro Nicaragüense" 1a ed. -Managua: Editarte, 2010. 126 p.

Pedroza, H. (2015) "Sistema de Innovación" 1a ed. -Managua: Editarte, 2015

Pedroza, H. (2016) "Conferencia Magistral sobre Innovación Organizacional” presentada en el curso de Gerencia de los Procesos de Inniovación y Emprendimiento

Pedroza, H. (2015) "Documento base Instalación SNIA" 1a ed. -- Managua: Editarte,

Roja J. y Espinoza E. (2013). "Desarrollo Rural Territorial": enfoque, metodologías y experiencias --1a ed. - Managua, 2013136 p. 